PROCEEDINGS OF THE AMERICAN MATHEMATICAL SOCIETY

Volume 124, Number 10, October 1996

\title{
SYMMETRIES OF PERIODIC SOLUTIONS FOR PLANAR POTENTIAL SYSTEMS
}

\author{
MARTIN GOLUBITSKY, JIAN-MIN MAO, AND MATTHEW NICOL
}

(Communicated by Hal L. Smith)

\begin{abstract}
In this article we discuss the symmetries of periodic solutions to Hamiltonian systems with two degrees of freedom in mechanical form. The possible symmetries of such periodic trajectories are generated by spatial symmetries (a finite subgroup of $\mathbf{O}(\mathbf{2})$ ), phase-shift symmetries (the circle group $\mathbf{S}^{1}$ ), and a time-reversing symmetry (associated with mechanical form). We focus on the symmetries and structures of the trajectories in configuration space $\left(\mathbb{R}^{2}\right)$, showing that special properties such as self-intersections and brake orbits are consequences of symmetry.
\end{abstract}

\section{INTRODUCTION}

In [4] the notion of admissibility for symmetries of attractors was introduced. Let $\Sigma$ be a subgroup of a finite group $\Gamma$ acting on $\mathbb{R}^{n}$. The subgroup $\Sigma$ is admissible if there is a $\Gamma$-equivariant mapping $f: \mathbb{R}^{n} \rightarrow \mathbb{R}^{n}$ with an attractor $A$ whose symmetries are exactly $\Sigma$. The question of admissibility for discrete dynamical systems is discussed in $[4,2]$ and for diffeomorphisms in [5]. A similar question can be asked in Hamiltonian systems and in time-reversible systems. In such systems, however, the notion of attractors must be replaced by stable invariant sets.

In this note we address one small aspect of this larger question: What are the possible symmetries of periodic solutions in time-reversible systems with two degrees of freedom? We classify these symmetries by the symmetries of the projected trajectory in configuration space. Montaldi, Roberts and Stewart [8] have addressed this and more general questions for normal modes — periodic solutions of Hamiltonian systems occuring near equilibria. Our results are stronger in that they are global, but weaker in that we have not indicated how to find solutions with these types in specific equations.

Our approach has important differences from the admissibility results considered in these previous works, and these differences simplify substantially the answers we find. In our work we consider only the symmetry type that periodic solutions can have when the group $\Gamma$ is a finite subgroup of $\mathbf{O}(\mathbf{2})$. Our classification of these symmetry types is obtained using only properties of reflections and rotations in $\mathbf{O}(\mathbf{2})$. As a result we do not answer the question of admissible subgroups for periodic solutions of a given $\Gamma$; rather we answer the question of finding all symmetry types

Received by the editors June 15, 1994.

1991 Mathematics Subject Classification. Primary 58F22, 34C25, 58F05.

Research supported in part by NSF Grant DMS-9101836, ONR Grant N00014-94-1-0317, and the Texas Advanced Research Program (003652037). 
for some $\Gamma \subset \mathbf{O}(\mathbf{2})$. In our approach, we view symmetries of periodic solutions as consisting of space symmetries (elements in $\Gamma$ ), phase-shift symmetries (in $\mathbf{S}^{1}$, see [6]), and time-reversing symmetries (using the standard time-reversing symmetry of second order equations). These symmetries of the Hamiltonian equations manifest themselves when viewing periodic trajectories in configuration space in several ways. There are spatial symmetries that fix the solution pointwise, and there are spatial symmetries that move the trajectory onto itself (without fixing every point on the trajectory) either by preserving the direction of the flow (phase-shift symmetries) or by reversing it (time-reversing symmetries). A primary complication in classifying symmetries of periodic solutions stems from distinguishing these various types of symmetry.

In Table 1 we list the 14 possible symmetry types of periodic solutions. In Figures 1-3 we illustrate each of the various symmetry types by schematic oriented trajectories in configuration space. In Figures 4 and 5 we illustrate some of these symmetry types by showing actual periodic solutions obtained by numerical integration of a $\mathbf{D}_{3}$-invariant Hamiltonian system. For each of the symmetry types $3 \mathrm{c}, 4 \mathrm{a}$ and $5 \mathrm{c}$ we give a quadratic Hamiltonian that has periodic solutions with this symmetry type, thus showing that periodic orbits of these types may be the solution curves of linear Hamiltonian vector fields.

Specifically, we consider Hamiltonian equations of the form

$$
\dot{x}=p \quad \text { and } \quad \dot{p}=-\frac{\partial V}{\partial x}(x),
$$

where $x, p \in \mathbb{R}^{n}$ and the Hamiltonian $H$ has the form

$$
H(x, p)=\frac{1}{2}|p|^{2}+V(x)
$$

Note that all solutions of this potential system have the form $X(t)=(x(t), \dot{x}(t))$ and that all such systems have time-reversing symmetries. In particular, define $(R \cdot X)(t)=(x(-t),-\dot{x}(-t))$. If $X$ is a solution, then so is $R \cdot X$.

We also assume that the Hamiltonian is invariant under the action of a compact Lie group $\Gamma \subset \mathbf{O}(\mathbf{n})$, that is, $H(\gamma x, \gamma p)=H(x, p)$ for all $\gamma \in \Gamma$. This is equivalent to requiring that the potential $V$ be group invariant, that is, $V(\gamma x)=V(x)$ for all $\gamma \in \Gamma$. Later we specialize to the case where $n=2$ and $\Gamma$ is a finite subgroup of $\mathbf{O}(\mathbf{2})$.

The spatial symmetry group of a solution $X(t)=(x(t), \dot{x}(t))$ in configuration space is defined as the subgroup

$$
T=\{\gamma \in \Gamma: \gamma\{x(t)\}=\{x(t)\}\} .
$$

In our discussions, we shall use only the facts that our systems are second order equations $(\ddot{x}=F(x, \dot{x}))$ which have a first integral, are time-reversible $(F(x,-y)=$ $F(x, y))$, and are $\Gamma$-equivariant $(F(\gamma x, \gamma y)=\gamma F(x, y)$ for all $\gamma \in \Gamma)$. We end this section by mentioning a few physically motivated examples.

Polar coordinates $(r, \theta)$ provide a simple way to write a $\mathbf{D}_{3}$-invariant system. In these coordinates the Hamiltonian has the form

$$
H=\frac{1}{2}\left(p_{r}^{2}+p_{\theta}^{2}\right)+V(r, \theta)
$$

where $\left(p_{r}, p_{\theta}\right)$ are the momentum coordinates and the potential $V(r, \theta)$ is given by

$$
V(r, \theta)=f(r, \theta)+f\left(r, \theta-\frac{2 \pi}{3}\right)+f\left(r, \theta-\frac{4 \pi}{3}\right),
$$


where $f(r, \theta)$ is an arbitrary function. Here are two examples.

(1) In a plane consider a mass being drawn by three identical springs of unit length, whose ends are fixed at the corners of an equilateral triangle. Then

$$
f(r, \theta)=-\frac{1}{2} k\left[\sqrt{1-2 r \cos (\theta)+r^{2}}-1\right]^{2},
$$

where $k$ is the force constant of the springs [3]. This example generalizes easily to give a $\mathbf{D}_{m}$-invariant example.

(2) Consider localized electrons in a two-dimensional hexagonal lattice of positive ions where electrons are placed in the neighborhood of the center of each triangle, and consider nearest-neighbor interaction. Under these assumptions we can consider the dynamics in each triangle separately, and

$$
f(r, \theta)=-\frac{\alpha}{\sqrt{1-2 r \cos (\theta)+r^{2}}},
$$

where $\alpha$ is the strength of interaction [1].

Finally, we mention a $\mathbf{D}_{2}$-invariant example: a hydrogen electron in a uniform magnetic field. In cylindrical coordinates $(r, \phi, z)$ the Hamiltonian is independent of $\phi$ and is given by

$$
H=\frac{1}{2}\left(p_{r}^{2}+p_{z}^{2}\right)-\frac{1}{\sqrt{r^{2}+z^{2}}}+\alpha r^{2},
$$

where $\alpha$ is a constant related to the strength of the magnetic field [7].

\section{Symmetries of Periodic solutions}

We suppose that $X(t)$ is a periodic solution with minimum period $2 \pi$, and show that the spatial symmetry group $T$ is obtained from a certain isotropy subgroup of $X$. Periodic solutions have three symmetry groups. We have described two previously: the space symmetries $\Gamma$ and the time-reversing symmetry $R$. The third one is the phase-shift symmetry group $\mathbf{S}^{1}$, where $\theta \in \mathbf{S}^{1}$ acts on $X$ by $\theta \cdot X(t)=$ $X(t+\theta)$. Since $\mathbf{S}^{1}$ and $R$ do not commute, they generate a group isomorphic to $\mathbf{O}(\mathbf{2})$. The full group of symmetries acting on periodic solutions is $\hat{\Gamma}=\Gamma \times \mathbf{O}(\mathbf{2})$. More precisely, if $(\gamma, \theta, \varepsilon) \in \hat{\Gamma}$ where $\varepsilon= \pm 1$, then

$$
(\gamma, \theta, \varepsilon) X(t)=(\gamma x(\varepsilon t+\theta), \varepsilon \gamma \dot{x}(\varepsilon t+\theta))
$$

is also a periodic solution of (1.1) with minimum period $2 \pi$.

Given the group action of $\hat{\Gamma}$ on $2 \pi$-periodic solutions, we can define the isotropy subgroup $\hat{\Sigma}$ of a given solution $X(t)$; that is,

$$
\hat{\Sigma}=\{(\gamma, \theta, \varepsilon) \in \hat{\Gamma}:(\gamma, \theta, \varepsilon) X=X\}
$$

Let $\Pi_{\Gamma}: \hat{\Gamma} \rightarrow \Gamma$ be projection. We prove the following:

Proposition 2.1.

$$
T=\Pi_{\Gamma}(\hat{\Sigma}) .
$$

This proposition states that the symmetry group $T$ of the periodic solution in configuration space is the projection of the isotropy subgroup $\hat{\Sigma}$ of the periodic solution in phase space. 
Proof. It follows directly from (2.1) that $\gamma\{x(t)\}=\{x(t)\}$; hence $T \supset \Pi_{\Gamma}(\hat{\Sigma})$.

To prove the converse, let $\tau \in T$; that is, let $\tau\{x(t)\}=\{x(t)\}$. Since $\tau x(0)=x(\theta)$ for some $\theta \in \mathbf{S}^{1}$, it follows that $\tau x(t)$ for $t$ near 0 lies on the curve $x\left(t^{\prime}\right)$ for $t^{\prime}$ near $\theta$. Hence $\tau \dot{x}(0)$ is a scalar multiple of $\dot{x}(\theta)$. Since the Hamiltonian $H$ must be constant on the trajectory $X(t)=(x(t), \dot{x}(t))$, it follows from (1.2) that $\tau \dot{x}(0)=\varepsilon \dot{x}(\theta)$, where $\varepsilon= \pm 1$. Uniqueness of solutions to systems of ODE now implies that $(\tau, \theta, \varepsilon)$ fixes

the solution $X$ and is in $\hat{\Sigma}$. Thus $\tau \in \Pi_{\Gamma}(\hat{\Sigma})$, as desired.

\section{Classifichtion of Symmetries}

Let $\hat{\Sigma}_{0}$ be the group of spatio-temporal symmetries of $X(t)$; that is, let

$$
\hat{\Sigma}_{0}=\hat{\Sigma} \cap\left(\Gamma \times \mathbf{S}^{1}\right) .
$$

Observe that $\hat{\Sigma}_{0}$ is a twisted subgroup, that is, there is a group homomorphism

$$
\Theta: T_{0} \rightarrow \mathbf{S}^{1},
$$

where $T_{0}=\Pi_{\Gamma}\left(\hat{\Sigma}_{0}\right)$, such that

$$
\hat{\Sigma}_{0}=\left\{(\gamma, \Theta(\gamma)) \in \Gamma \times \mathbf{S}^{1}: \gamma \in T_{0}\right\}
$$

This follows from uniqueness of solutions to systems of ODE.

To summarize: $T$ is the subgroup of symmetries acting on configuration space which leave the periodic trajectory $\{x(t)\}$ invariant; $T_{0} \subset T$ is the subgroup consisting of symmetries that preserve the direction of time; and $\operatorname{ker} \Theta \subset T_{0}$ is the subgroup consisting of symmetries that fix the trajectory $x(t)$ pointwise.

In this note we address the question: What are the possible symmetry subgroup triplets $\left(T, T_{0}\right.$, $\left.\operatorname{ker} \Theta\right)$ when $X$ is a periodic orbit? We simplify this question by classifying the symmetries of periodic solutions up to conjugacy in $\mathbf{O}(\mathbf{2})$ rather than up to conjugacy in the space group $\Gamma \subset \mathbf{O}(\mathbf{2})$ - as is often done. We prove the following:

Theorem 3.1. Up to conjugacy in $\mathbf{O}(\mathbf{2}) \times \mathbf{O}(\mathbf{2})$, the symmetries of periodic solutions are given in Table 1.

Before proceeding with the proof we define a special class of periodic solutions defined by their trajectories in configuration space. A periodic solution is a brake orbit if the trajectory $x(t)$ in configuration space lies on a smooth curve with endpoints. This result, stated in a slightly different form, may be found in [8], Remark (IV), p. 707 .

Lemma 3.2. A trajectory $X(t)$ is a brake orbit if and only if $X(t)$ has a phase-shift $X(t+\theta)$ with time-reversing symmetry $(I, 0,-1)$.

Proof. If $X(t)$ is a $2 \pi$-periodic solution that has $(I, 0,-1)$ as a symmetry, then $x(-t)=x(t)$. It follows that the trajectory $x(t)$ runs from $x(0)$ to $x(\pi)$ and then retraces its path back to $x(0)$. (Note that $x(0) \neq x(\pi)$; otherwise, uniqueness of solutions would imply that $X$ is $\pi$-periodic.) Thus $X$ is a brake orbit.

Conversely, suppose that $X(t)=(x(t), \dot{x}(t))$ is a brake orbit. Phase shift $X$ so that $x(0)$ is an endpoint; thus $\dot{x}(0)=0$. The form of (1.1) implies that $Y(t)=$ $(x(-t),-\dot{x}(-t))$ is also a solution. However, the initial conditions for $X$ and $Y$ at $t=0$ in phase space are the same. Hence $X$ and $Y$ are identical, and $(I, 0,-1)$ is a symmetry of $X$. 
TABle 1. Possible symmetries for periodic solutions: $\tau, \tau_{1}$, and $\tau_{2}$ denote reflections.

\begin{tabular}{|c|c|c|c|c|c|c|}
\hline Name & $T$ & $T_{0}$ & $\operatorname{ker} \Theta$ & reversing & reference & features \\
\hline (1) & $\overline{\mathbf{D}_{k}(k \geq 3)}$ & $\overline{\mathbb{Z}_{k}}$ & 1 & $(\tau, 0,-1)$ & (b2) & \\
\hline$\overline{(2)}$ & $\overline{\mathbb{Z}_{k}(k \geq 3)}$ & $\mathbb{Z}_{k}$ & 1 & none & (a) & \\
\hline (3a) & $\overline{\overline{\mathbf{D}_{2}}}$ & $\overline{\mathbb{Z}_{2}}$ & $\overline{11}$ & $\overline{(\tau(\tau, 0,-1)}$ & $\overline{(\overline{b 2})}$ & \\
\hline (3b) & $\mathbf{D}_{2}$ & $\mathbf{D}_{2}$ & $\mathbf{D}_{1}$ & $(I, 0,-1)$ & (b1) & brake \\
\hline$(3 \mathrm{c})$ & $\mathbf{D}_{2}$ & $\mathbf{D}_{1}\left(\tau_{1}\right)$ & 1 & $\left(\tau_{2}, 0,-1\right)$ & (b2) & intersecting \\
\hline$(4 a)$ & $\mathbf{D}_{1}$ & $\mathbf{D}_{1}$ & 1 & none & (a) & intersecting \\
\hline$(4 b)$ & $\mathbf{D}_{1}$ & $\mathbf{D}_{1}$ & 1 & $(I, 0,-1)$ & (b1) & brake \\
\hline$(4 \mathrm{c})$ & $\mathbf{D}_{1}$ & $\mathbf{D}_{1}$ & $\mathbf{D}_{1}$ & $(I, 0,-1)$ & (b1) & brake \\
\hline$(4 \mathrm{~d})$ & $\mathbf{D}_{1}$ & 1 & 1 & $(\tau, 0,-1)$ & (b2) & \\
\hline$(5 \mathrm{a})$ & $\overline{\mathbb{Z}_{2}}$ & $\overline{\mathbb{Z}_{2}}$ & $\overline{1}$ & none & (a) & \\
\hline$(5 \mathrm{~b})$ & $\mathbb{Z}_{2}$ & $\mathbb{Z}_{2}$ & 1 & $(I, 0,-1)$ & (b1) & brake \\
\hline$(5 \mathrm{c})$ & $\mathbb{Z}_{2}$ & 1 & 1 & $(-I, 0,-1)$ & (b2) & intersecting \\
\hline$(6 a)$ & 1 & 1 & 1 & none & (a) & \\
\hline (6b) & 1 & 1 & 1 & $(I, 0,-1)$ & (b1) & brake \\
\hline
\end{tabular}

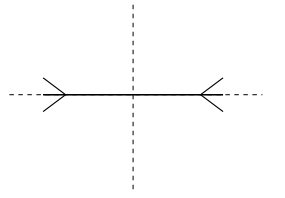

(3b)

$$
\mathrm{R}=(\mathrm{I}, 0,-1)
$$

(4b)

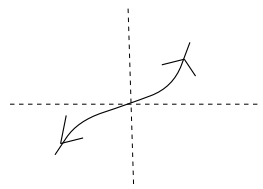

(5b)

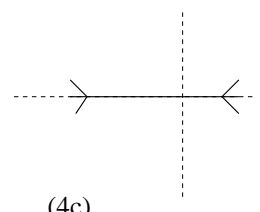

(4c)
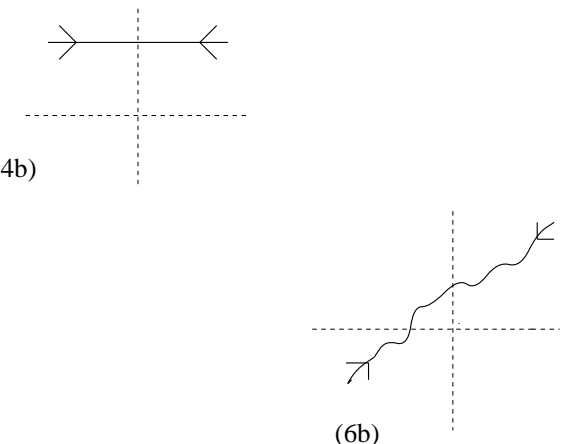

(6b)

Figure 1. Configuration space illustrations of brake orbits. Dotted lines indicate coordinate axes. 


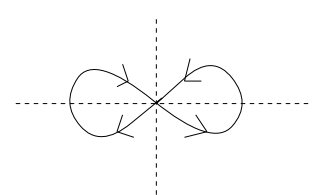

$(3 \mathrm{c})$

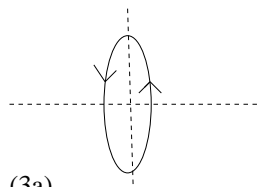

(3a)
$\mathrm{R}=(\tau, 0,-1)$

(1)

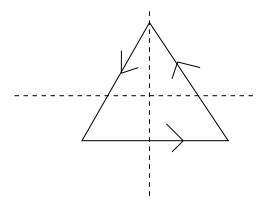

(4d)

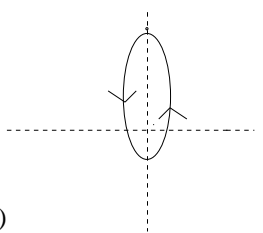

Figure 2. Configuration space illustrations of trajectories having time-reversing symmetry $(\tau, 0,-1)$

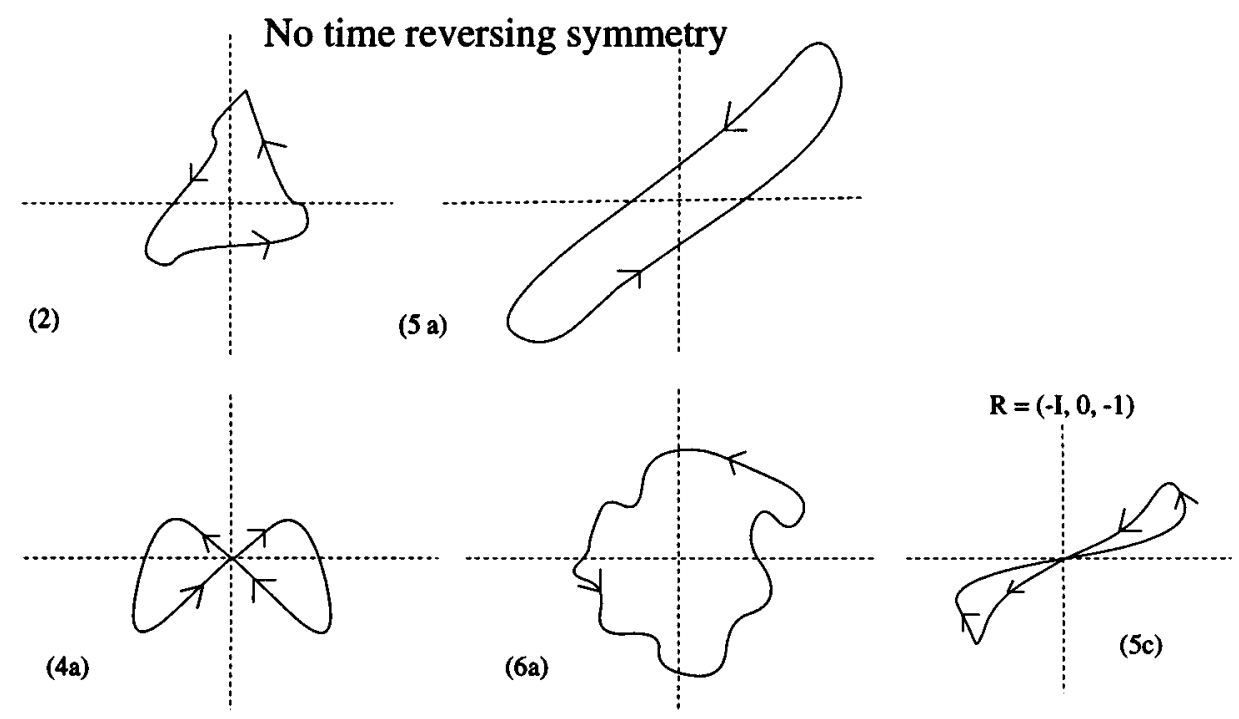

FigurE 3. Configuration space illustrations of the remaining trajectories 

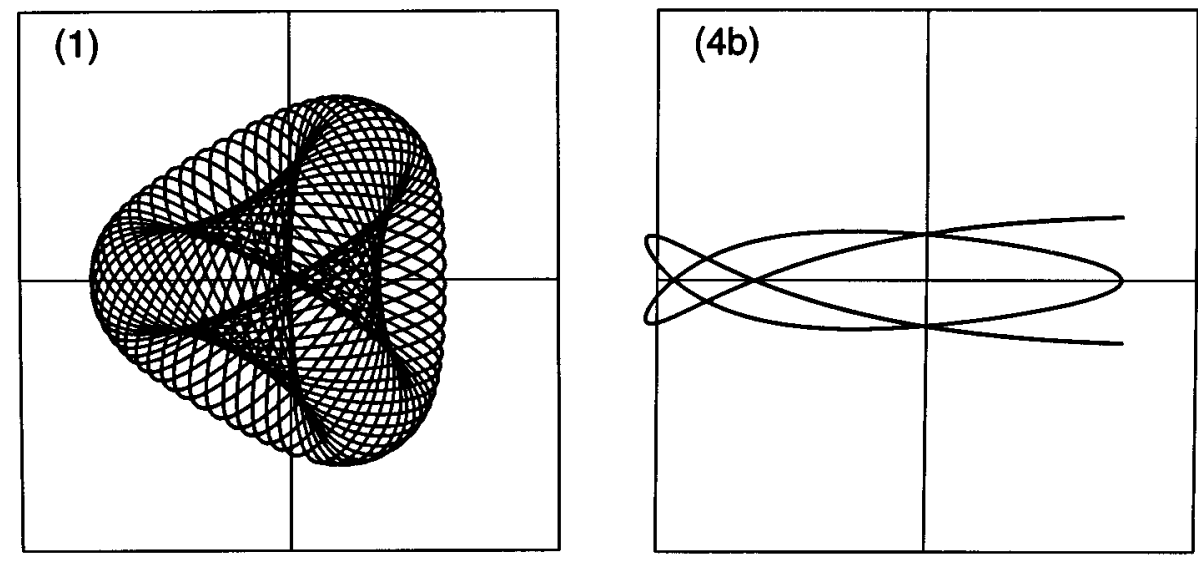

Figure 4. Type (1) trajectory: $V(x, y)=\frac{1}{2}\left(x^{2}+y^{2}\right)+\frac{2}{3}\left(x^{3}-3 x y^{2}\right)$ with energy $\frac{1}{49.9}$; initial conditions $(0.0,0.0,0.00895,0.2)$. Type (4b) trajectory: $V(x, y)=\frac{1}{2}\left(x^{2}+y^{2}\right)+\frac{2}{3}\left(x^{3}-3 x y^{2}\right)$ with energy $\frac{1}{32}$; initial conditions $(0.0,0.052,0.2416,0.038)$.

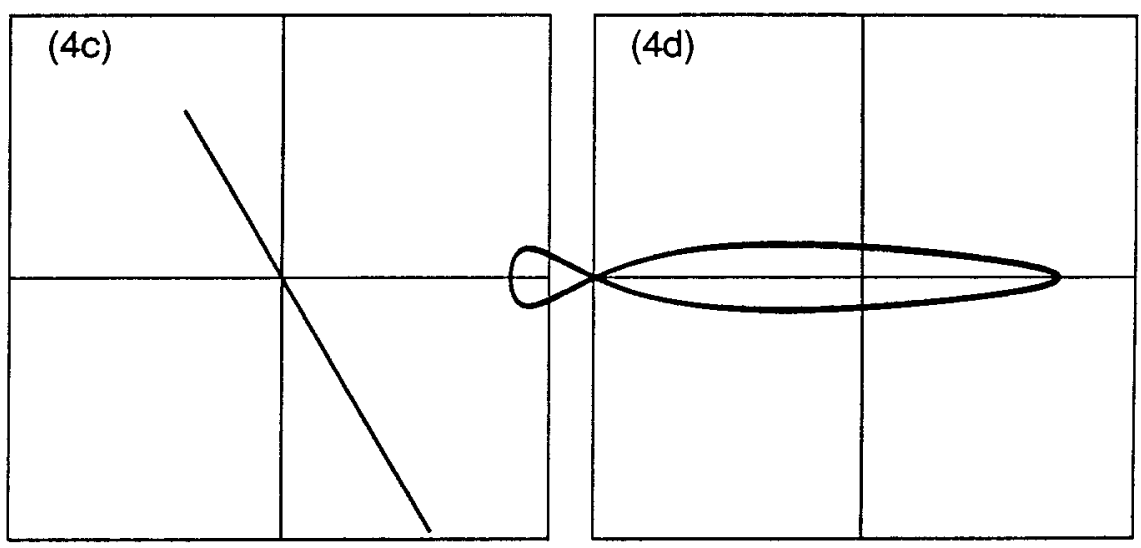

Figure 5. Type (4c) trajectory: $V(x, y)=\frac{1}{2}\left(x^{2}+y^{2}\right)+\frac{2}{3}\left(x^{3}-\right.$ $\left.3 x y^{2}\right)$ with energy $\frac{1}{32}$; initial conditions $(0.0,0.0,0.125,-0.2165)$. Type (4d) trajectory: $V(x, y)=\frac{1}{2}\left(x^{2}+y^{2}\right)+\frac{2.28}{3}\left(x^{3}-3 x y^{2}\right)$ with energy $\frac{1}{32}$; initial conditions $(0.0,0.011,0.2497,-0.0055)$. 
Proof of Theorem 3.1. It follows from (3.1) that $\operatorname{ker} \Theta$ is a normal subgroup of $T_{0}$ and that $T_{0} / \operatorname{ker} \Theta$ is cyclic. Note that the elements of $\operatorname{ker} \Theta$ fix $x(t)$ pointwise, so that $x(t) \in \operatorname{Fix}(\operatorname{ker} \Theta)$.

Since $\Gamma \times \mathbf{S}^{1} \subset \hat{\Gamma}$ is of index two, either $\hat{\Sigma}=\hat{\Sigma}_{0}$ or $\left|\hat{\Sigma} / \hat{\Sigma}_{0}\right|=2$. In the former case $T=T_{0}$ is immediate, while in the latter case there are two possibilities: $T=T_{0}$ and $\left|T / T_{0}\right|=2$. Altogether, the symmetry group $T$ may be constructed in one of three ways from $\hat{\Sigma}$ as follows:

(a) $\hat{\Sigma}=\hat{\Sigma}_{0}$

(b1) $\left|\hat{\Sigma} / \hat{\Sigma}_{0}\right|=2$ and $T=T_{0}$, or

(b2) $\left|\hat{\Sigma} / \hat{\Sigma}_{0}\right|=2$ and $\left|T / T_{0}\right|=2$.

We consider each of these possibilities in turn.

First, we specialize to the two degrees of freedom case and assume that $\Gamma \subset \mathbf{O}(\mathbf{2})$ is a finite group. Since $x(t)$ is not constant, it follows that the only possibilities for $\operatorname{ker} \Theta$ are the trivial group $\mathbf{1}$ and the two element reflection group $\mathbf{D}_{1}$. If $\operatorname{ker} \Theta=\mathbf{D}_{1}$, then the trajectory $x(t)$ must lie in the line of symmetry of $\mathbf{D}_{1}$ and hence be a brake orbit. Lemma 3.2 implies that after a phase shift we may assume that $(I, 0,-1)$ is a symmetry; hence $\left|\hat{\Sigma} / \hat{\Sigma}_{0}\right|=2$. Since $T_{0} / \operatorname{ker} \Theta$ is cyclic, the only possibilities for $T_{0}$ are $T_{0}=\mathbf{D}_{1}$ or $T_{0}=\mathbf{D}_{2}$.

If $\operatorname{ker} \Theta=\mathbf{1}$, then either $T_{0}=\mathbb{Z}_{k}$ for some $k \geq 1$, or $T_{0}=D_{1}$.

(a) $\hat{\Sigma}=\hat{\Sigma}_{0}$.

The assumption $\hat{\Sigma}=\hat{\Sigma}_{0}$ implies that $X(t)$ has no time-reversing symmetry. If $\operatorname{ker} \Theta=\mathbf{D}_{1}$, then $\{x(t)\}$ is a brake orbit and by Lemma 3.2 has a time-reversing symmetry, contradicting the assumption. Thus $\operatorname{ker} \Theta=\mathbf{1}$ and $T$ is cyclic. Hence $T=\mathbb{Z}_{k}$ for some $k \geq 1$ (case 2 when $k \geq 3$, case 5 a when $k=2$, and case 6 a when $k=1)$, or $T=D_{1}$ (case $\left.4 \mathrm{a}\right)$.

(b1) $\left|\hat{\Sigma} / \hat{\Sigma}_{0}\right|=2$ and $T=T_{0}$.

The assumption $\left|\hat{\Sigma} / \hat{\Sigma}_{0}\right|=2$ implies that $X(t)$ has a time-reversing symmetry, and the assumption $T=T_{0}$ implies that this time-reversing symmetry has the form $(I, \theta,-1) \in \hat{\Gamma}$. After phase shifting we may assume that $X(t)$ has the time-reversing symmetry $(I, 0,-1)$. Lemma 3.2 implies that these trajectories are brake orbits.

Should $\operatorname{ker} \Theta=\mathbf{D}_{1}$, then the trajectory is an interval in the line of symmetry of $\mathbf{D}_{1}$. Thus $T=\mathbf{D}_{2}$ if that interval is symmetric about the origin (case $3 \mathrm{~b}$ ), and $T=\mathbf{D}_{1}$ otherwise (case $4 \mathrm{c}$ ).

If $\operatorname{ker} \Theta=\mathbf{1}$, then the trajectory $x(t)$ is a planar curve with two distinct endpoints $x(0)$ and $x(\pi)$. Note that the symmetry group $T$ must preserve endpoints. If a symmetry $\tau \in T$ fixes these two endpoints, then $\tau$ must be a reflection and, by uniqueness of solutions, $x(t)$ lies in $\operatorname{Fix}(\tau)$. It follows that $\operatorname{ker} \Theta \neq \mathbf{1}$, which is a contradiction. Thus, all nontrivial elements in $T$ must interchange endpoints, and it follows that $T$ has order two. Hence $T=\mathbf{1}$ (case $6 \mathrm{~b}$ ), $T=\mathbf{D}_{1}$ (case $4 \mathrm{~b}$ ), or $T=\mathbb{Z}_{2}$ (case $\left.5 \mathrm{~b}\right)$.

(b2) $\left|\hat{\Sigma} / \hat{\Sigma}_{0}\right|=2$ and $\left|T / T_{0}\right|=2$.

As noted previously, the only possibilities for $\operatorname{ker} \Theta$ are $\mathbf{1}$ and $\mathbf{D}_{1}$. However if $\operatorname{ker} \Theta=\mathbf{D}_{1}$, then the trajectory is a brake orbit and has been classified in the previous subsection. So $\operatorname{ker} \Theta=\mathbf{1}$, and $T_{0}$ must be cyclic. There are three cases:

$(\alpha) T_{0}=\mathbb{Z}_{k}$ for some $k \geq 2$ (cases 1 and $\left.3 \mathrm{a}\right)$,

(ß) $T_{0}=\mathbf{1}$ (cases $4 \mathrm{~d}$ and $5 \mathrm{c}$ ), or

$(\gamma) T_{0}=\mathbf{D}_{1}$ (case 3c), 
In case $(\alpha)\left|T / T_{0}\right|=2, T$ may be either $T=\mathbf{D}_{k}$ (cases 1 and $3 a$ ) or $T=$ $\mathbb{Z}_{2 k}$. In fact, $T=\mathbb{Z}_{2 k}$ cannot occur when $k \geq 2$. To verify this point, note that $\Pi_{\Gamma}\left(\hat{\Sigma}-\hat{\Sigma}_{0}\right)=T-T_{0}$. Hence the periodic solution $X(t)$ must have a symmetry of the form $(\gamma, \theta,-1)$, where $\gamma$ is rotation by $\frac{\pi}{k}$. It follows that $\left(\gamma^{2}, 0,1\right)$ is a symmetry of $X(t)$, and hence that $\gamma^{2}$ fixes $x(t)$ pointwise. Since $\gamma^{2}$ is a rotation, $\gamma^{2}=1$. Hence $T=\mathbb{Z}_{2 k}$ is impossible when $k \geq 2$.

In case $(\beta)$ we have $T_{0}=\mathbf{1}$ and two possibilities for $T$; namely, $T=\mathbb{Z}_{2}$ (case $5 \mathrm{c}$ ) and $T=\mathbf{D}_{1}$ (case $\left.4 \mathrm{~d}\right)$. Case $(\gamma)$ leads directly to $T=\mathbf{D}_{2}$ (case $\left.3 \mathrm{c}\right)$.

Suppose that a periodic solution has a projection in configuration space with $\mathbf{D}_{k}$ symmetry where $k \geq 3$. Then Theorem 3.1 implies that the reflectional symmetries in $\mathbf{D}_{k}$ must be time-reversing.

\section{TRAJECTORY GEOMETRY}

Lemma 3.2 shows that a trajectory is a brake orbit if and only if (a phase-shift conjugate of ) the trajectory has $(I, 0,-1)$ as a time-reversing symmetry. We remark that for non-brake orbits self-intersection points are sometimes forced by symmetry. In particular, we prove the following:

Proposition 4.1. Let $X(t)$ be a periodic trajectory that is not a brake orbit. If either

(a) $(\tau, \theta, 1)$ is a space symmetry where $\tau$ is a reflection or

(b) $(-I, 0,-1)$ is a time-reversing symmetry of $X$,

then $X$ has a self-intersection point in configuration space.

In case (a) there must be a self-intersection on the line of symmetry of $\tau$, while in case (b) there must be a self-intersection point at the origin.

Proof. Suppose that $(\tau, \theta, 1)$ is a space symmetry of $X=(x, \dot{x})$. Since $\left(\tau^{2}, 2 \theta, 1\right)$ $=(1,2 \theta, 1)$ is also a space symmetry, either $\theta=0$ or $\theta=\pi$. Note that $(\tau, 0,1)$ is a space symmetry if and only if $x(t) \in \operatorname{Fix}(\tau)$. Therefore, if $\theta=0$, then $X(t)$ is a brake orbit; so we may assume $\theta=\pi$. Symmetry and continuity imply that $\{x(t)\} \cap \operatorname{Fix}(\tau) \neq \emptyset$ - say, $x\left(t_{0}\right) \in \operatorname{Fix}(\tau)$. The symmetry $(\tau, \pi, 1)$ implies that $x\left(t_{0}\right)=x\left(t_{0}+\pi\right)$, which shows that there is a self-intersection.

Suppose that $(-I, 0,-1)$ is a symmetry of $X$. Then $x(-t)=-x(t)$ and $x(0)=0$. Similarly, $x(\pi)=x(-\pi)=-x(\pi)$ so that $x(\pi)=0$, showing that $x$ goes through the origin at two different times.

\section{Constructions of trajectories}

Proposition 4.1 implies that trajectories of type 3c, 4a and $5 \mathrm{c}$ must have selfintersections. It can be verified that each of these symmetry types occurs in periodic solutions of linear Hamiltonian equations. See Table 2. We assume that the Hamiltonian has the form

$$
H(x, y, p, q)=\frac{1}{2}\left(|p|^{2}+|q|^{2}\right)+V(x, y) .
$$

In Figures 1-3 we draw plausible sketches for each of the possible symmetry types. In these drawings we show that for each of the symmetry types that are not brake orbits and are not covered by Proposition 4.1, there are possible trajectories $x(t)$ without self-intersections. In Figures 4 and 5 we show numerical solutions for several symmetry types. 
TABLE 2. Periodic solutions with self-intersections.

\begin{tabular}{|c|c|c|}
\hline$V(x, y)$ & solution & type \\
\hline$\frac{x^{2}}{2}+2 y^{2}$ & $(\sin (t), \sin (2 t), \cos (t), 2 \cos (2 t))$ & $3 \mathrm{c}$ \\
$\frac{5}{4}\left(x^{2}+y^{2}\right)-\frac{3}{2} x y$ & $(\sin (t)+\sin (2 t), \sin (t)-\sin (2 t)$, & $5 \mathrm{c}$ \\
& $\cos (t)+2 \cos (2 t), \cos (t)-2 \cos (2 t))$ & \\
$2 x^{2}+\frac{1}{2} y^{2}$ & $(\sin (2 t), 2 \cos (t)+\sin (t), 2 \cos (2 t),-2 \sin (t)+\cos (t))$ & $4 \mathrm{a}$ \\
\hline
\end{tabular}

\section{ACKNOWLEDGMENT}

We thank Ian Stewart, James Montaldi, and Jerry Marsden for a number of helpful comments.

\section{REFERENCES}

[1] N.W. Ashcroft and N.D. Mermin. Solid State Physics, W.B. Saunders, Philadelphia, 1976.

[2] P. Ashwin and I. Melbourne. Symmetry groups of attractors. Arch. Rat. Mech. Anal. 126 (1994) 59-78. MR 95a:58077

[3] A.D. Boardman, D.E. O'Connor and P.A. Young. Symmetry and its Applications in Science, McGraw-Hill, London, 1973.

[4] I. Melbourne, M. Dellnitz and M. Golubitsky. The structure of symmetric attractors, Arch. Rational Mech. \&f Anal. 123 (1993) 75-98. MR 94m:58141

[5] M. Field, I. Melbourne and M. Nicol. Symmetric attractors for diffeomorphisms and flows. Proc. Lond. Math. Soc. 72 (1996), 657-696.

[6] M. Golubitsky and I. N. Stewart, Hopf bifurcation in the presence of symmetry. Arch. Rational Mech. Anal. 87 No. 2 (1985) 107-165. MR 86g:58034

[7] J.M. Mao and J.B. Delos. Hamiltonian bifurcation theory of closed orbits in diamagnetic Kepler problem. Phys. Rev. A 45 (1992) 1746-1761.

[8] J. Montaldi, M. Roberts and I. Stewart. Existence of nonlinear normal modes of symmetric Hamiltonian systems I, Nonlinearity 3 (1990) 695-730. MR 92e:58190

Department of Mathematics, University of Houston, Houston, Texas 77204-3476

E-mail address: mg@uh.edu

Department of Mathematics, Hong Kong University of Science and Technology, HONG KONG

E-mail address: mamao@uxmail.ust.hk

Department of Mathematics, UMist, Manchester, United Kingdom

E-mail address: m.nicol@umist.ac.uk 